\title{
Extra-Osseous Osteochondroma of the Knee
}

\author{
P Priya J, MD Patho, M Valiathan, MD Patho, L Rao, MD Patho \\ Department of Pathology, Kasturba Medical College, Manipal, India
}

\begin{abstract}
A 39 year old male presented with complaints of ongoing pain and restricted movements of right knee joint for a period of 7 years. Plain radiographs showed a calcified mass in the popliteal fossa. Open excision and biopsy was performed, and microscopic examination revealed features of extraosseous osteochondroma. Integrated clinical-pathologic diagnosis protocols are helpful in ascertaining the nature of extraskeletal cartilaginous tumours that arise at unusual anatomic sites. Complete local surgical excision is the management of choice.
\end{abstract}

Key Words:

Extra-osseous, Osteochondroma, Knee

\section{INTRODUCTION}

Osteochondromas usually develop in relation to the periosteum, and occur around the growth plate of long bones, especially around the knee. These tumours usually stop growing with closure of the growth plate, but some continue to grow after skeletal maturity. Intra-articular osteochondromas are rare in older individuals. In joints with a large capsular space, such as the patellofemoral joint, osteochondromas can remain intra-articular. Overall behaviour of extraskeletal osteochondromas is poorly characterised ${ }^{1}$.

Osteochondromas are often confused with giant synovial chondromatosis or the more dangerous chondrosarcomas ${ }^{2}$. Detailed histological analysis is important to rule out malignant changes. The distinction between these growths is important to avoid radical surgery, as osteochondromas are benign lesions with a good prognosis after local excision.

\section{CASE HISTORY}

A 39 year old male presented with complaints of ongoing pain and restricted movements of right knee joint for the previous 7 years. Range of flexion gradually decreased over the 7 year time period. Pain was nonradiating in nature, located mainly in the posterior aspect of the right knee and calf, without any diurnal variation and present only on terminal flexion. On palpation, the back of the knee showed a swelling of approx $7 \times 7 \mathrm{~cm}$ in the right popliteal fossa with the skin over swelling being normal. There was also mild suprapatellar fullness noted on the left side along with synovial hypertrophy.

Radiographs of the right knee showed a calcified mass in the popliteal fossa (Figure 1). There were multiple nodular cartilaginous calcifications seen along the posterior aspect of the knee joint, representing synovial osteochondromatosis. The lesion was completely extraarticular with no connection to the underlying bone. As the radiological picture was suggestive of a benign lesion, an open excision and biopsy of the mass was performed. Postoperatively, the patient's knee was kept at 20 degrees of flexion using an immobilizer. Gross surgical examination revealed multiple glistening nodular tissue fragments with grey brown and hemorrhagic areas, with the largest fragment measuring $7 \times 5 \times 1.5 \mathrm{cms}$. Microscopically, the lesion was composed of a mature hyaline cartilaginous cap overlying lamellar bony trabeculae, enclosing fatty marrow spaces (Figure 2). A patchy area of dense sclerosis in bone was also seen. A diagnosis of extraosseous osteochondroma was made.

\section{DISCUSSION}

Solitary synovial osteochondroma is a rare variant of synovial osteochondromatosis ${ }^{1}$. These tumours arise from the synovial cells/ primitive cells resident in the synovium. The tumours are formed by the metaplasia of synovial cells to chondrocytes, giving rise to islands of cartilage ${ }^{3}$. Solitary synovial osteochondroma is not connected to the bone and occurs in the juxta-articular regions of the small joints of hand and feet. These lesions may be large, and mimic the clinical and radiological features of a malignant process. Osteochondromas found within the soft tissue without osseous or intra-articular involvement are more accurately described as extraskeletal osteochondromas. They are slowgrowing, benign, osseous tumours, and are often misdiagnosed as conventional osteochondromas. Though extraskeletal osteochondromas are rare, they typically affect the digits of the hands or feet ${ }^{4}$.

A review of literature shows variable nomenclature used for such lesions including, extra-osseous osteochondroma-like soft tissue mass ${ }^{1}$, solitary synovial osteochondroma ${ }^{3}$, 


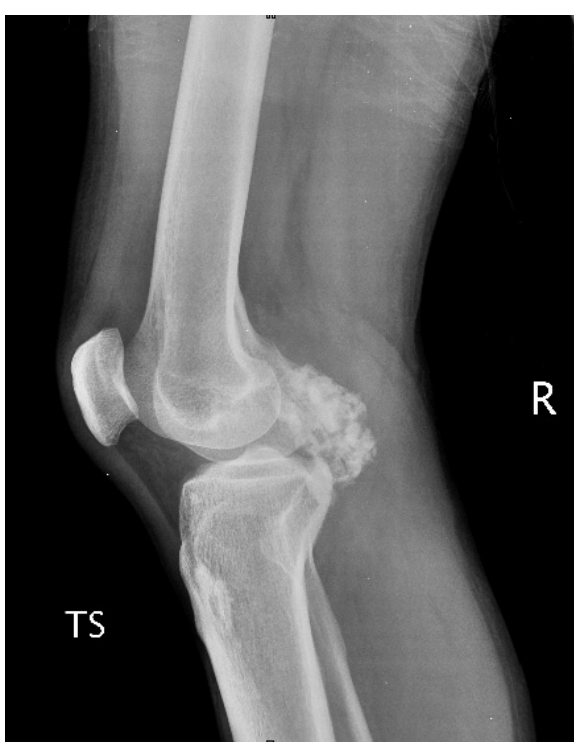

Fig. 1: X-ray of the right knee showing a calcified mass in the popliteal fossa, with multiple nodular cartilaginous calcifications along the posterior aspect of knee joint. The lesion is completely extra-articular with no connection to the underlying bone.

extraskeletal osteochondroma ${ }^{4}$, para-articular/ extra-osseous osteochondroma $^{5}$ and giant extraskeletal osteochondroma (of the foot). Clear histological features indicating the origin of an osteochondroma are not available in the present case. Given the presence of the cartilage-free area of the pedicle, the osteochondroma may have originated at the posterior end plate of the tibia or femur, and grown towards the articular cavity of the knee. The pedicle may have then been torn so that the extra-osseous osteochondroma-like soft tissue mass became truly loose within the knee joint. When calcifications or bone formations are visible on standard X-rays, differential diagnosis should include consideration of soft tissue tumours with calcification such as liposarcoma or synovial sarcoma, popliteal aneurysm, arteriovenous malformation, synovial chondromatosis and post-traumatic loose bodies.

The most serious threat in the presence of an osteochondroma is malignant transformation of the

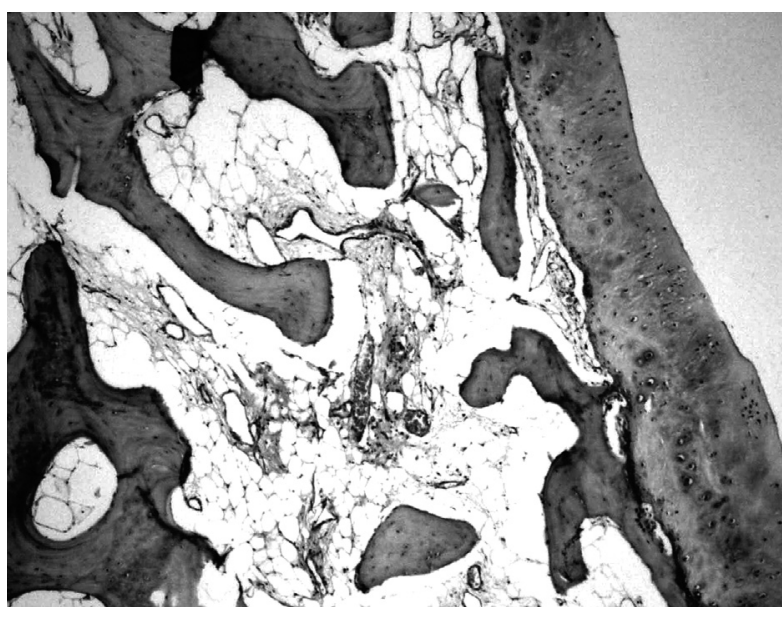

Fig. 2: Microphotograph showing mature hyaline cartilaginous cap overlying lamellar bony trabeculae, enclosing fatty marrow spaces. (H\&E x100).

cartilaginous cap into a chondrosarcoma. A radiological feature consistent with such a transformation is an increase in the soft tissue mass over the bony component of the osteochondroma. Thickness of more than $2 \mathrm{~cm}$ of the extraosseous component on axial CT or MR images has been correlated with malignant transformation in adults. Sarcomatous degeneration of osteochondral lesions such as synovial chondromatosis may be easier to identify through careful histological examination and correlation with radiographic and operative findings.

\section{ACKNOWLEDGEMENTS}

We would like to thank Dr. Sripathi Rao, Dean and Professor, Department of Orthopaedics, Kasturba Medical College, Manipal and faculty, Department of Radiology, for providing us with the clinical details and radiological images. 


\section{REFERENCES}

1. Oliva F, Marconi A, Fratoni S, Maffulli N. Extra-osseous osteochondroma-like soft tissue mass of the patello-femoral space. BMC Musculoskelet Disord. 2006; 7: 57.

2. Dhillon MS, Prasad P, Goel A, Kar A. Giant osteochondral body in a popliteal cyst. Indian J Orthop. 2009; 40(1): 93-6.

3. Allahabadia VN, Kulkarni SS, Dogra AS, Bhosale PB. Solitary synovial osteochondroma of the Knee. J. Postgrad Med. 1995; 41(4): 113-4.

4. Sheff JS, Wang S. Extraskeletal osteochondroma of the foot. J Foot \& Ankle Surg. 2005; 44(1): 57-9.

5. Dhillon MS, Sharma R, Vaiphei K, Nagi ON. Para-articular/ extra-osseous osteochondroma of the knee. The Knee. 1999; 6(2): 155-8. 